\title{
ASTRONOMY FROM A LUNAR BASE
}

\author{
S. VOLONTE \\ European Space Agency \\ 8-10 rue Mario Nikis \\ 75738 Paris Cedex 15 \\ France
}

\begin{abstract}
The Moon is generally considered to be an ideal site for astronomy, offering excellent observing conditions and access to the entire electromagnetic and particle spectrum. A wide range of astronomical observations can be carried out from the Moon, but, as concluded in a recent ESA study (Mission to the Moon 1992), only a restricted number could be better implemented from a lunar site rather than from any other location. Very low frequency (VLF) astronomy, astrometry and interferometry fall into this category, as well as a transit telescope to map dark matter in the Universe. Whilst VLF and astrometric telescopes should be automatic, long baseline interferometers will probably require human intervention and will thus benefit from a manned lunar base.
\end{abstract}

\section{Rationale for astronomy from the Moon}

The Moon is regarded as an attractive site for astronomy, offering significant advantages over ground based observatories. It is very stable with only low levels of seismicity. Its attitude is very well known. The absence of atmosphere gives access to the entire electromagnetic and particle spectrum and, moreover, the far side is free from electromagnetic interferences.

Some of these advantages are also common to free flyers but the main benefits from the Moon are its mechanical and thermal stability and its large size giving the possibility of extendability and serviceability. There are some drawbacks such as micrometeorite impacts causing damage to instruments, pollution due to naturally levitated dust (or caused by vehicle or man made activities), large temperature variations between night and day, important thermal radiation from the lunar soil and no access to the full celestial sphere. But, a major problem is the considerably higher cost of Moon based instruments compared to orbiting instrumentation.

Nevertheless, in the context of a significant lunar programme (including manned activities) synergies could develop between lunar exploration missions and astronomical observations (e.g. sharing of power resources, communication, etc.), which would favour the development of almost any kind of astronomical application (Mumma and Smith 1990). Cost implications make it imperative that Moon based observations offer essential, if not unique, advantages over any other observational locations. Based on such considerations, a recent ESA study (Misson to the Moon 1992) has concentrated on three types of instrumentation for a lunar implementation; Radiotelescopes for Very Low Frequency (VLF) astronomy; Instruments for high precision astrometry, including Earth-Moon VLBI; Imaging interferometers for observation from UV to submillimeter wavelengths. More recently a lunar transit telescope has also been proposed as an attractive lunar instrument to search for dark matter in the Universe.

\section{VLF Astronomy}

VLF represents the extreme low energy part of the electromagnetic spectrum, corresponding to decametric $(10-15 \mathrm{MHz})$ and longer wavelengths. It is an almost unexplored window which would 
allow to access the lowest practicable physical limit to study the radiation from as diverse objects as the Earth itself, the Sun, the giant planets, the spiral arms of own and other normal galaxies. Due to ionospheric absorption, VLF is practically unobservable from the ground except occasionally at a few favourable locations. Full exploration of this domain therefore awaits deployment in space and an ideal location would be the lunar far side free from electromagnetic interferences.

The resolution imposed by the angular size of discrete radio sources calls for large apertures achievable by interferometry. But interplanetary and interstellar scattering limit the ultimate angular resolutions from a few degrees to a few tens of arcsec translating into baselines from one to hundreds of kilometers. On the Moon, such VLF systems could consist of arrays of antenna deposited on the far side, connected to light, low-power receivers working at low data rate, allowing to carry out searches for extra-solar planets around nearby stars, as well as extending VLF astronomy towards extragalactic targets.

\section{Optical Astrometry}

The high angular resolution achievable by interferometry provides a most attractive capability for the future development of astrometry. In principle microarcsec accuracies can be achieved with baselines extending from $100 \mathrm{~m}$ to $1 \mathrm{~km}$ and faint magnitudes $\left(\mathrm{m}_{\mathrm{v}} \sim 20\right)$ can be reached with 1 $\mathrm{m}$-class telescopes. The scientific case for astrometry at this level is very strong indeed (Ridgway 1993) and the the Moon would appear as a suitable platform for this class of astrometric measurements. However, regarding global astrometry, interferometric measurement of large angles require either very large delay lines and movable telescopes, or re-orientation of the whole instrument. Since these motions must be controlled to an accuracy of a few nanometres and full sky visibility is needed, the Moon does not seem to be a convenient site for global astrometry which, indeed, is best achieved from free flyers.

For small angle measurements (small angle astrometry) of e.g. parallaxes and proper motions relative to background quasars, internal motions of clusters and nearby galaxies, search for planets, etc., the Moon, with its well known slow and smooth rotation could be useful. For instance, a fixed east-west interferometer with short delay lines could observe the transit of objects across a strip of e.g. 10 arcmin along the local meridian, providing one dimensional coordinate difference tied to the local lunar rotation rate. However, the sky coverage would be very limited and no astrophysically useful proper motions would be provided unless several different baseline orientations would be possible. Thus small angle astrometry from the Moon would be of restricted application even with very long baselines and several possible orientations.

\section{Earth - Moon very long baseline interferometry (VLBI)}

A single radio telescope located on the Moon, working in conjunction with ground telescopes would permit VLBI with a single baseline of some $400000 \mathrm{~km}$. Since the Moon's distance and position can be determined accurately to a few $\mathrm{mm}$ and milliarcsecs respectively, the Earth-Moon VLBI system would be particularly suited for very accurate astrometry. The dramatic increase in angular resolution would allow, for instance, to build a network of reference extragalactic sources to unprecendented accuracies (microarcsecs).

However such baselines limit detection to sources with high brigthness temperatures and interstellar scattering increases the apparent size of objects with wavelength, thus imposing the use of large high frequency antennae. Nevertheless, a precursor system could use a relatively low frequency single medium class antenna $(\sim 10 \mathrm{~m})$ with a passively cooled receiver. The 
implementation of a more ambitious system would be dictated by transportation and construction issues.

\section{Lunar Interferometry}

The ultimate goal in modern astronomy is to reach diffraction limited imaging at all wavelengths for objects of small angular sizes. However, in the UV to submillimeter range the Earth's atmosphere either absorbs radiation (partially or totally) or its fluctuations restrict the spatial resolution $(\sim 1$ arcsec in the visible). Techniques now exist (speckle interferometry, adaptive optics) which allow to approach the theoretical resolving power of ground based filled apertures. But the ultimate resolutions are reached with interferometers which combine coherently the signals of several telescopes. For ground based interferometers, the atmosphere limits the sensitivity because large, individual apertures are illuminated coherently only over small areas. In space, full sensitivity can be achieved at once but the apertures will be smaller. The Moon therefore seems to offer a significant potential as it combines most of the advantages of ground based and space borne observations.

The scientific case for lunar interferometry has been extensively made (Mission to the Moon 1992, ESA Interferometry Review Panel 1994). In the optical range, the long term goal is to provide for imaging at kilometric baselines with angular resolution better than 100 microarcsec. An imaging interferometer requires more than two telescopes to obtain good quality images and allow phase closure techniques to be applicable. The Moon's slow rotation rate imposes a sufficient number of telescopes in the system to obtain adequate uv plane coverage within a reasonable exposure time. In addition, long optical delay lines are required to compensate for the pathlength differences due to this rotation. This is indeed, the most difficult problem of a Moon interferometer because weak signals might need to travel over long distances close to the Moon's surface, possibly through a thin atmosphere of electrostatically levitated dust. The complexity of the system increases with the number of telescopes, which in turn, will remain limited, thus requiring the telescopes to be movable to provide the required spatial frequency coverage. In other words, a imaging optical interferometric array will be an extremely complex instrument and the need to deploy it on the Moon cannot be evaluated on scientific grounds alone. The choice will be dictated by technical and cost considerations related in particular to deployment and transportation issues.

In the infrared and submillimetre range, since the Earth atmosphere is almost completely opaque to this radiation, the issue here is the choice of the best location in space, the Moon being a possible site. The long term goal is to provide imaging at high spatial resolution in both the lines and continuum. This will be limited by sensitivity requirements imposing use of large antennas (a few meters in diameter). Sensitivity will also restrict the achievable angular resolution to about 0.1 arcsec even for the brightest sources thus limiting the baselines (a few tens to a few hundred meters) but favouring good imaging. So for this wavelength domain sensitivity requirements impose a large total collecting area necessitating large structures and probably deployable antennas. From the science standpoint, it is not obvious without further evidence, to conclude that a Moon based submillimetre interferometer would offer any definitive advantages over a free flying instrument. In both cases, the instrument would be very complex.

\section{Lunar Transit Telescope}

Fort and co-workers (Fort and Mellier 1994) have recently proposed to use the dense population of background galaxies as a reference grid at large distance to look at the statistical gravitational 
image distortions to detect the contours and masses of foreground structures. In other words, when the light emitted from distant sources passes near large deflecting masses, the beams are weakly bent thus introducing in the cross section a small gravitational ellipticity perpendicular to the projected gravity field.

On the ground, this effect is detected up to amplitudes of $~ 1-5 \%$ allowing to study dense halos of dark matter around clusters and groups of galaxies. Extrapolation to the largest scale structures of the Universe requires to detect amplitudes of about $3.10^{-4}$ with a spatial resolution of about one degree. Even with the best seeing conditions, instrumental distortions of ground based observations cannot be reliably calibrated to this level due to a variety of reasons (minute mechanical flexures, tracking jitter, fast seeing changes and atmospheric refraction effects). The Moon, with its lower gravity and its stable, slow rotation rate would allow to overcome these problems with a fixed $1 \mathrm{~m}$ transit telescope ( 0.5 to 1 degree field of view) observing long strips of the sky with a large CCD mosaic. Preliminary simulations indicate that this technique would allow to reach the required accuracy. In addition, such a telescope could be used for deep surveys at high spatial resolution and it could also be seen as an automatic precursor telescope of more elaborate interferometric instruments.

\section{Conclusions}

Although almost any kind of astronomical observations could be carried out from the Moon, only a restricted number could make best use of the Moon environment. As outlined in this paper, some ambitious projects can and probably will, at some stage, be implemented on the Moon. The complex, long duration observations required by these lunar instruments will be more efficiently implemented for remote, automatic use. However, human intervention is expected to be requested in the deployment and maintenance of such complex facilities as e.g. large interferometric systems. Potential nuisances due to human presence and related activities (vehicles, etc.) will have to be limited as much as possible. In particular the lunar far side will need to be protected from electromagnetic pollution.

\section{$\underline{\text { References }}$}

ESA Interferometry Review Panel, 1994, Report, in preparation Fort B. and Mellier Y., 1994, Astron. \& Astrophys. Review, in press

Mission to the Moon, 1992, ESA SP-1150

Mumma M.J., Smith H.J., Editors, 1990 : Astrophysics from the Moon, American Institute of Physics, Conference Proceedings 207, New York

Ridgway S., 1993, The scientific support for a space interferometry mission; Spaceborne Interferometry, SPIE Vol. 1947, Reasenberg R.D., Editor 